
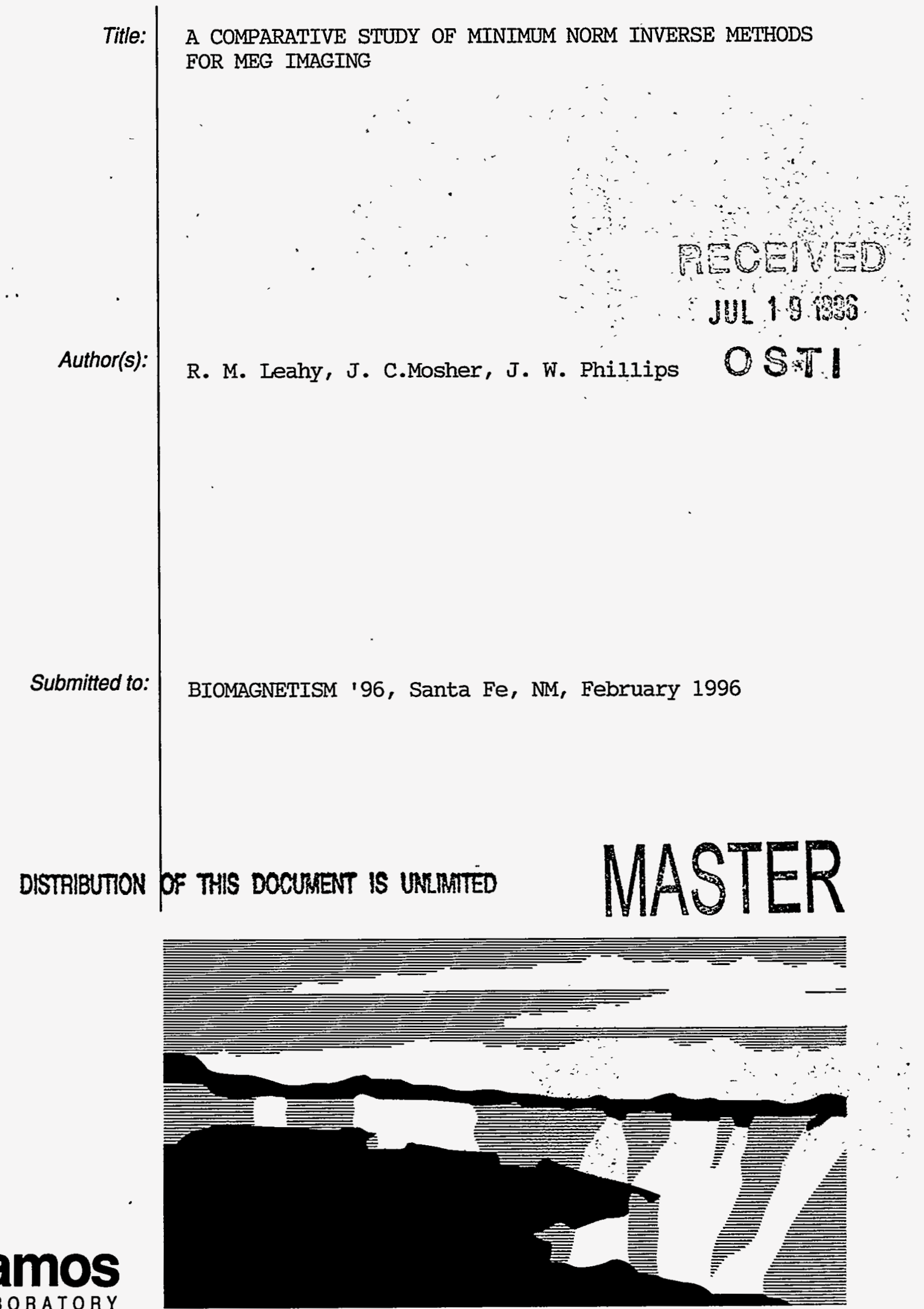

Los Alamos National Laboratory, an affirmative action/equal opportunity emplóyer, is operated by the University of California for the U.S. Department of Energy under contract W-7405-ENG-36. By acceptance of this article, the publisher recognizes that the U.S. Government retains a nonexclusive, royalty-free license to publish or reproduce the published form of this contribution, or to allow others to do so, for U.S. Government purposes. The Los Alamos National Laboratory requests that the publisher identify this article as work performed under the auspices of the U.S. Department of Energy. 


\section{DISCLAIMER}

This report was prepared as an account of work sponsored by an agency of the United States Government. Neither the United States Government nor any agency thereof, nor any of their employees, makes any warranty, express or implied, or assumes any legal liability or responsibility for the accuracy, completeness, or usefulness of any information, apparatus, product, or process disclosed, or represents that its use would not infringe privately owned rights. Reference herein to any specific commercial product, process, or service by trade name, trademark, manufacturer, or otherwise does not necessarily constitute or imply its endorsement, recommendation, or favoring by the United States Government or any agency thereof. The views and opinions of authors expressed herein do not necessarily state or reflect those of the United States Government or any agency thereof. 


\title{
A Comparative Study of Minimum Norm Inverse Methods for MEG Imaging ${ }^{\dagger}$
}

\author{
${ }^{1}$ Leahy, R.M., ${ }^{2}$ Mosher, J.C., and ${ }^{1}$ Phillips, J.W.

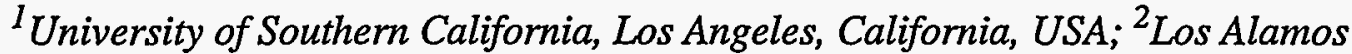 \\ National Laboratory, Los Alamos, New Mexico, USA
}

\begin{abstract}
The majority of MEG imaging techniques currently in use fall into the general class of (weighted) minimum norm methods. The minimization of a norm is used as the basis for choosing one from a generally infinite set of solutions that provide an equally good fit to the data. This ambiguity in the solution arises from the inherent non-uniqueness of the continuous inverse problem and is compounded by the imbalance between the relatively small number of measurements and the large number of source voxels. Here we present a unified view of the minimum norm methods and describe how we can use Tikhonov regularization to avoid instabilities in the solutions due to noise. We then compare the performance of regularized versions of three well known linear minimum norm methods [5][7] with the non-linear iteratively reweighted minimum norm method [1] and a Bayesian approach described in our companion paper ("MEG-based Imaging of Focal Neuronal Current Sources," Phillips J.W., Leahy R.M., Mosher J.C.).
\end{abstract}

\section{The Minimum Norm Approach to MEG Imaging}

Since the forward model in MEG is linear, we may relate the $N$ sources $y(N \times 1)$ and the $M$ MEG measurements $\mathbf{b}(M \times 1)$ as $\mathbf{b}=\mathbf{G y}+\mathbf{n}$, where the $i^{\prime}$ th row of the $M \times N$ system matrix $\mathbf{G}$ is a discrete representation of the lead field (sensitivity) of the $i$ 'th sensor. The $j$ 'th column of $\mathbf{G}$ specifies the gain vector for the $j^{\prime}$ th constrained dipole component. The MxI vector $\mathbf{n}$ represents noise generated within the sensor and by unwanted electromagnetic sources.

Since the inverse problem is inherently ill-posed, the search for an appropriate imaging method is concerned with finding a way to choose within a set of images that produce essentially the same fit to the data. The weighted minimum $\mathrm{l}_{2}$-norm approach to linear inverse problems involves solving the constrained optimization problem:

$$
\mathbf{y}_{\mathrm{wmn}}=\underset{\mathrm{y}}{\arg \min } \mathbf{y}^{\mathrm{T}} \mathbf{C}_{\mathbf{y}}^{-1} \mathbf{y} \text { such that }|\mathbf{b}-\mathbf{G y}|^{2}=0
$$

where $C_{y}$ is an arbitrary symmetric positive definite matrix. Writing $C_{y}=W W^{T}$, we can form the solution as,

$$
y_{w m n}=W W^{T} G^{T}\left(G W W^{T} G^{T}\right)^{-1} b=W(G W)^{t} b
$$

where $(\mathbf{G W})^{\dagger}$ denotes the pseudoinverse of $\mathbf{G W}$. The weighted minimum norm solution can also be formed from the singular value decomposition (SVD) of GW :

$$
\mathbf{y}_{\mathrm{wmn}}=\mathrm{W} \sum_{i=1}^{M}\left(\frac{\mathbf{u}_{i}^{\mathrm{T}} \mathbf{b}}{s_{i}}\right) \mathbf{v}_{i}
$$

where $s_{i}, \mathbf{v}_{i}$, and $\mathbf{u}_{i}$ are the $i$ 'th singular value and corresponding right and left singular vectors of $\mathbf{G W}$, respectively.

Several forms of $W$ have been proposed for MEG imaging applications. In [6] and [7], the weight matrix is implicitly the identity matrix (W=1). In the normalized minimum norm method [4], $\mathbf{W}_{\text {norm }}=\operatorname{diag}\left(1 /\left\|\mathbf{g}_{1}\right\|, 1 /\left\|\mathbf{g}_{2}\right\| \ldots, 1 /\left\|\mathbf{g}_{N}\right\|\right)$, where $\left\|\mathbf{g}_{i}\right\|$ is the Euclidean norm of the $i$ 'th column of $\mathbf{G}$. This weighting is designed to compensate for the reduced sensitivity of MEG to deep sources resulting in a preference for superficial distributions when $\mathrm{W}=\mathrm{I}$. The $N \times N$ Laplacian operator $\mathbf{B}$ is commonly selected to smooth the reconstruction in minimum norm imaging, with $W=B^{-1}$. The LORETA technique [5] uses a weighting matrix $W_{L}=W_{\text {norm }} B^{-1}$. The Laplacian operator tends to select smoother reconstructions than either of the previous two weight functions.

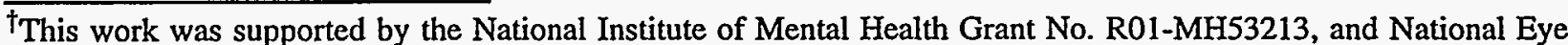
Institute Grant No. R01-EY08610-04. 
Exact matching of the data in (1) results in ill-conditioning and high sensitivity to noise. Regularized forms of weighted minimum norm methods lower this noise sensitivity. One popular technique is to simply truncate the summation in (3) at a threshold index. An alternative method, Tikhonov regularization [3], replaces the original problem (1) with the unconstrained minimization of a combination of the residual error norm and weighted $\mathrm{l}_{2}$-norm of the solution vector:

$$
y_{\lambda}=\underset{y}{\arg \min }|\mathbf{b}-\mathbf{G} \mathbf{y}|^{2}+\lambda \mathbf{y}^{\mathrm{T}} \mathbf{C}_{\mathbf{y}}^{-1} \mathbf{y} .
$$

For any value of the regularization parameter $\lambda$, the solution can be found as:

$$
\nabla_{\lambda}=\mathrm{WW}^{\mathrm{T}} \mathbf{G}^{\mathrm{T}}\left(\mathbf{G W} \mathrm{W}^{\mathrm{T}} \mathbf{G}^{\mathrm{T}}+\lambda \mathrm{I}\right)^{-1} \mathbf{b}=\mathrm{W} \sum_{i=1}^{M} f_{i}\left(\frac{\mathbf{u}_{i}^{\mathrm{T}} \mathbf{b}}{s_{i}}\right) \mathbf{v}_{1}
$$

where the filter parameters $f_{i}=s_{i}^{2} /\left(s_{i}^{2}+\lambda\right)$. Note that the filter coefficients decrease as $s_{i}$ decreases. Therefore, the contributions of $\left(\mathbf{u}_{i}^{\mathrm{T}} \mathbf{b} / s_{i}\right) \mathbf{v}_{i}$ to the solution from the smaller $s_{i}$ are effectively filtered out. In our implementation of the regularized weighted minimum norm methods we use a regularization toolbox [3] which selects an optimal value for $\lambda$ using the L-curve method.

The iteratively reweighted minimum norm approach, also known as focal underdetermined system solution (FOCUSS)[1], is a novel inverse method which iteratively updates the weight matrix using the result of the previous iteration. The algorithm first provides an initial estimate of $y(0)$ using (2) with $\mathbf{W}_{\text {norm }}$ as the weighting matrix. At each step thereafter, a separate weight matrix $W(k)=\operatorname{diag}(y(k-1))$ is formed. At each iteration, we update the solution using,

$$
\mathbf{y}(k)=\left(\mathbf{W}_{\text {norm }} \mathbf{W}(k)\right)\left(\mathbf{G} \mathbf{W}_{\text {norm }} \mathbf{W}(k)\right)^{\dagger} \mathbf{b} .
$$

For any starting point $y(0)$ asymptotic convergence to a fixed point is guaranteed [1]. The fixed points are, however, unknown and highly dependent on the initial estimate. Also, the final error in fitting the data may be large, even for the noiseless case. In our implementation we use Tikhonov regularization and the L-curve method [3] at each iteration to avoid ill-conditioning.

\section{The New Bayesian Approach}

We have developed a new approach to MEG imaging. We use a Bayesian formulation of the inverse problem in which a Gibbs prior is constructed to reflect our specific expectations regarding the spatial distribution of sources. The reconstruction method uses this prior to resolve ambiguities that are inherent in the inverse problem. A thorough description of this Bayesian technique is given in our companion paper ("MEG-based Imaging of Focal Neuronal Current Sources," Phillips, J.W., Leahy, R.M., and Mosher, J.C.).

Basic studies of functional activation, such as somatotopic or retinotopic mapping using fMRI or PET, reveal the sparse and highly localized nature of activation in the cerebral cortex. Our Gibbs prior is therefore specifically designed to reflect the expectation that current sources tend to be sparse and focal. We combine this with a Gaussian distribution for active sources and an assumption of additive Gaussian noise to form the posterior distribution. An estimate of the primary current source distribution for a specific data set is formed by maximizing over the posterior probability.

\section{Simulations}

We have conducted extensive simulations based on a simplified $2 D$ source model with a $1 D$ array of 64 sensors. All sources are constrained to the annular segment of the $x-y$ plane shown in Fig. 1 . The distance between source locations was set to $2.8 \mathrm{~mm}$ providing an image grid of 560 pixels. All dipoles were constrained in orientation perpendicular to the $x-y$ plane. The source images were chosen using stochastic sampling from the prior distribution on the image. The results of one representative

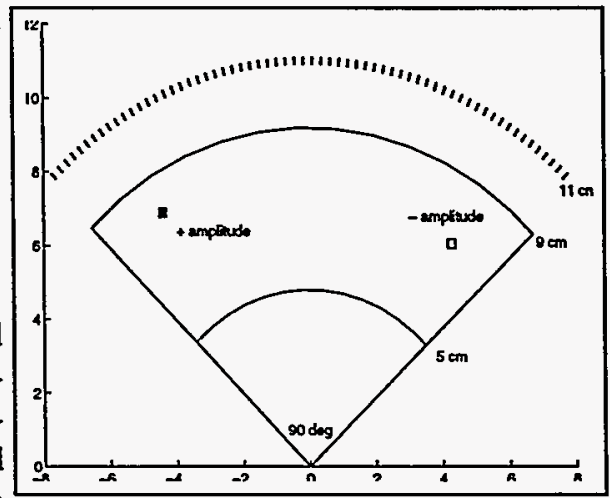

Fig. 1. Sample plot of one simulation solution showing representative positive and negative sources. 
simulation are shown in Fig. 2 for two scenarios, one noiseless, and one with added white Gaussian noise. We compared the Bayesian technique to the methods previously discussed: the standard minimum $\mathrm{l}_{2}$ norm technique, the normalized minimum norm technique, the LORETA method, and the iteratively reweighted minimum norm technique.

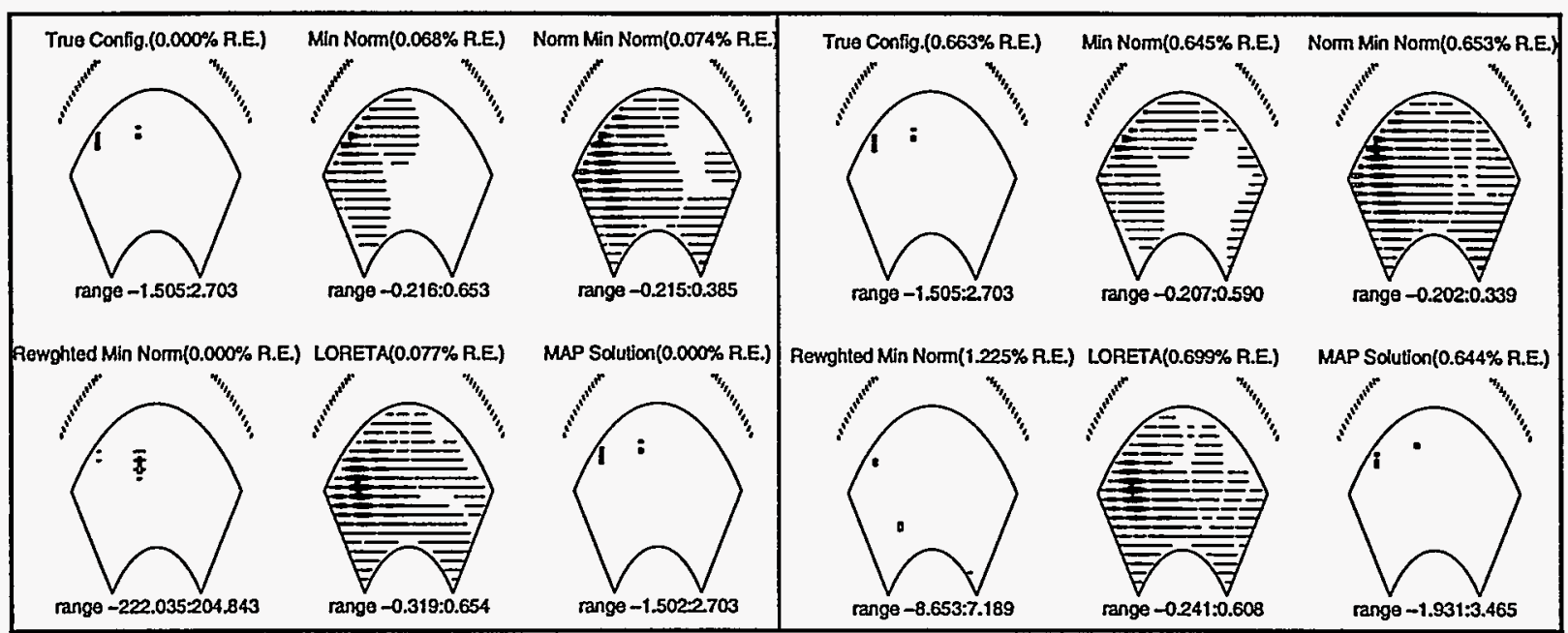

Fig. 2. Sample 2D simulation with a $1 D$ sensor array. 64 point measurements, 560 point image grid. The percent residual error (\% R.E.) is shown above and the range of source values (in nAm) is shown below each image. Two scenarios are presented: (left) No noise added to the computed data (right) White Gaussian noise added to the data to achieve a SNR of $20 \mathrm{~dB}$

\section{Phantom Experiments}

We have applied the same techniques used in the simulations to experimental phantom data collected with a Neuromag-122 system [2] using the manufacturer's 26 dipole phantom. The Neuromag-122 system employs 61 dúal-channel planar first-order gradiometer units in a helmetshaped configuration at a radius of $10-11 \mathrm{~cm}$, measuring the magnetic field gradient in two orthogonal tangential directions, for a total of 122 individual sensor measurements.

The phantom consists of two half circles with a 7 $\mathrm{cm}$ radius in the $\mathrm{x}-\mathrm{z}$ plane and $\mathrm{y}-\mathrm{z}$ plane, with dipoles in fixed positions in these planes oriented tangential to the outer edge. The image reconstruction grid consisted of 768 locations spaced $4 \mathrm{~mm}$ apart on two 180 degree annuli, with an inner radius of $3 \mathrm{~cm}$ and an outer radius of $7 \mathrm{~cm}$. An example of the imaging surface with the surrounding sensor elements is shown in Fig. 3. The distance between sources and sensors creates a gain matrix which is more poorly conditioned than the one in the previous simulations, which increases noise sensitivity. In all cases, the gain matrix was constructed to include gradiometer effects and non-radial

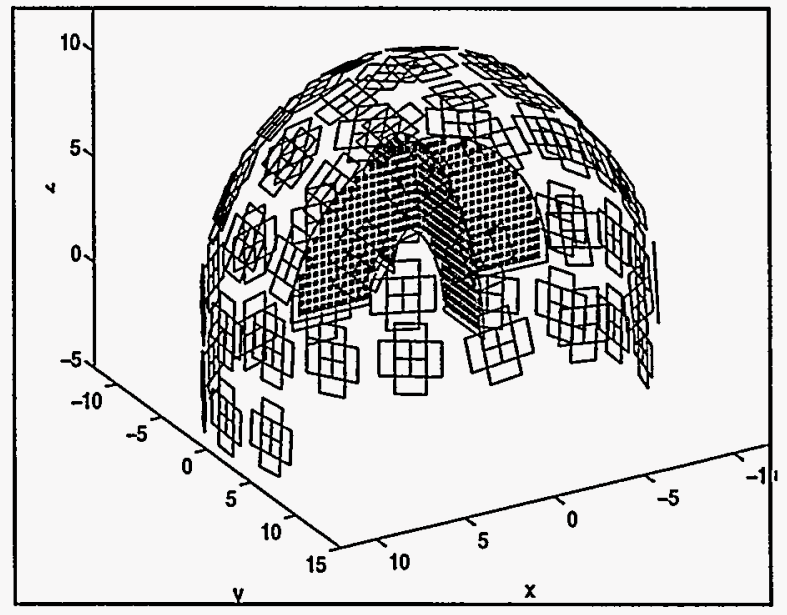

Fig. 3. Schematic representation of the phantom reconstruction regions (two orthogonal, 180 degree annular regions) with the locations of the planar gradiometers of the neuromag- 122 system superimposed. sensor orientations assuming a spherical source volume [6].

The phantom data was scaled to reflect a reasonable evoked field response. We then added data collected in the same system from a passive human subject (100 averages of a pre-stimulus interval from an evoked response paradigm). This background was added to the phantom data to obtain a specified SNR. Fig. 4 shows a comparison plot in which noise was added to obtain a SNR of $10 \mathrm{~dB}$. The minimum norm and Bayesian techniques tested on phantom data performed comparably to the simulations. 


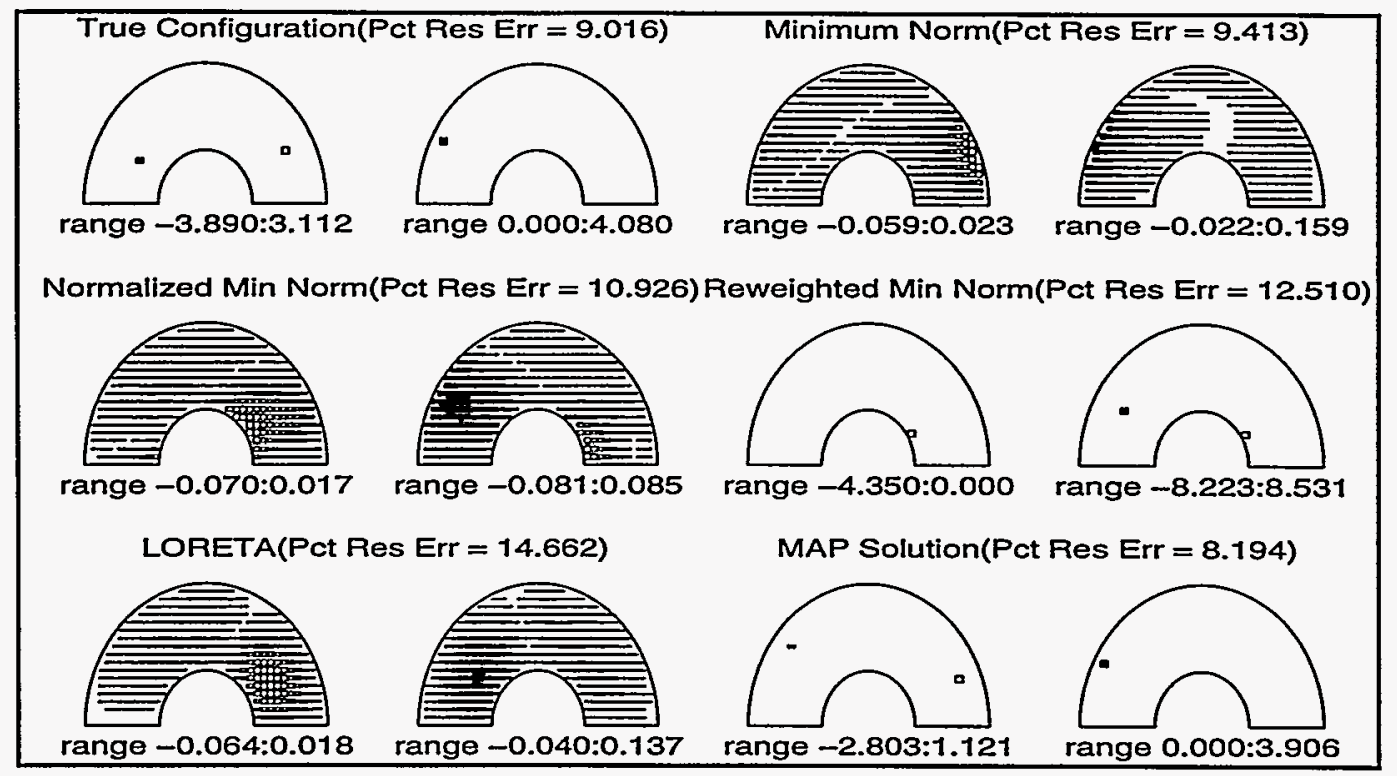

Fig. 4. Sample phantom results with three active dipoles on two orthogonal planes. 122 point measurements, 768 point image grid. Percent residual error (Pct Res Err) is shown above and the range of source values (in $\mathrm{nAm}$ ) is shown below each image. Colored subject noise added to the data to achieve a SNR of $10 \mathrm{~dB}$.

\section{Conclusions}

These results show a wide variation in the characteristics of the solution obtained using different weighting functions. All linear minimum norm methods produce results exhibiting a relatively large degree of smoothness. In comparison, both iteratively re-weighted minimum norm and the new Bayesian method produce very sparse solutions. For both simulations and the phantom experiment, all methods generally give similar residual errors in the fit to the data. In some instances, the iteratively reweighted minimum norm method gives larger errors since there is nothing inherent in the method to limit increases in the error from one iteration to the next. All results can be considered 'correct' in the sense that they are configurations that could have produced the observed data. This observation emphasizes the severely under-determined nature of this problem. Clearly, in order to select between these feasible solutions we must use additional information concerning the expected nature of the source. Our Bayesian approach specifically introduces the information that sources are sparse and focal into the reconstruction method which results in generally superior results for sources that exhibit these characteristics.

\section{References}

[1] I.F. Gorodnitsky, J.S. George, and B.D. Rao, "Neuromagnetic source imaging with FOCUSS: a recursive weighted minimum norm algorithm," EEG and clinical Neurophysiol., 95, pp. 231-251, 1995.

[2] M. Hämäläinen, R. Hari, et al., "Magnetoencephalography - theory, instrumentation, and applications to noninvasive studies of the working human brain," Rev Mod Phys, vol. 65(2), pp. 413-497, 1993.

[3] P.C. Hansen, Regularization Tools, A Matlab Package for Analysis and Solution of Discrete Ill-Posed Problems, available in Postscript from http://www.mathworks.com/, March 1993

[4] B. Jeffs, R.M. Leahy, and M. Singh, "An evaluation of methods for neuromagnetic image reconstruction," IEEE Trans on Bio. Eng, vol. 34, pp. 713-723, 1987.

[5] R.D. Pascual-Marqui, C.M. Michel, D. Lehmann, "Low resolution electromagnetic tomography: A new method for localizing electrical activity in the brain," Int. Journ. of Psyc., vol. 18, pp. 49-65, 1994

[6] J. Sarvas, "Basic mathematical and electromagnetic concepts of the biomagnetic inverse problem," Physics in Medicine and Biology, vol. 32, pp. 11-22, 1987.

[7] J.Z. Wang, S.J. Williamson, and L. Kaufman, "Magnetic Source Images Determined by Lead-Field Analysis: The Unique Minimum-Norm Least-Squares Estimation," IEEE Trans Bio. Eng., vol. 39(7), pp. 665-675, 1992. 